Pacific

Journal of

Mathematics

ASYMPTOTIC BEHAVIOUR OF EIGENFUNCTIONS ON SEMI-HOMOGENEOUS TREE

P. GÉRARdin AND K.F. Lai

Volume $196 \quad$ No. 2

December 2000 


\title{
ASYMPTOTIC BEHAVIOUR OF EIGENFUNCTIONS ON SEMI-HOMOGENEOUS TREE
}

\author{
P. GÉRARDin AND K.F. LAI
}

\begin{abstract}
A function on a tree is said to be harmonic if it is fixed under the averaging operators. We construct an explicit asymptotic formula giving the boundary distribution which reproduces the value of a harmonic function on a semi-homogeneous the tree.
\end{abstract}

\section{Introduction.}

The isomorphism from the space of hyperfunctions on the unit circle in the complex plane to the space of harmonic functions on the open unit disc can be given explicitly via the Poisson kernel. The analogue of the open unit disc over a non-archimedean local field is a tree. We prove an explicit asymptotic formula giving the boundary distribution which reproduces the value of an harmonic function on a semi-homogeneous tree.

The analogue over a nonarchimedean local field of the real Laplacian operator is the average operator (\$1.3). A function on a tree is said to be harmonic if it is fixed under the average operators. Given any harmonic function $f$ on a homogeneous tree $X$, Gerardin stated in $[\mathbf{G}] \S 2.4$ an explicit asymptotic formula giving the boundary distribution which reproduces the value of $f$ at a vertex $a$ of the tree. This covers the case of the BruhatTits building of the group $S L(2, F)$. For detailed discussion of this result and generalizations to eigenspaces of Laplacian on homogeneous trees using difference equations see Cohen-Colonna $([\mathbf{C C}])$. In this paper we generalize the above formula to the case of a semi-homogeneous tree (for example the Bruhat-Tits building of a unitary group) by solving an initial value problem of a difference equation.

Let $X$ be a semi-homogeneous tree with degree function $q$ (see $\S 1.1$ ). For any given vertex $a \in X$ the boundary $\Xi$ of $X$ is the inverse limit of the spheres $S(a, n)$ over $n \in \mathbb{N}$. The projection $\Xi \longrightarrow S(a, n)$ defines a map from the space $\mathcal{E}(X)$ of functions on $X$ to the space $\mathcal{D}(\Xi)$ of locally constant functions on $\Xi$ which we denote by $f \mapsto f_{a, n}$. The group $\Sigma(X)$ of characters of $X$ acts on the space $\mathcal{D}(\Xi)$; we write this action as $\varphi \mapsto \varphi^{s}$ (see $\S 2$ ). Let $d_{a}$ be the probability measure $d_{a}$ on $\Xi$ invariant under the stabilizer of $a$ in Aut $X$. The ratio $d_{b} / d_{a}$ is a function in $\mathcal{D}(\Xi)$. For each character $s \in \Sigma(X)$, 
we define the space $\mathcal{B}_{s}(\Xi)$ of maps $a \mapsto T_{a}$ from $X$ to the space $\mathcal{D}^{\prime}(\Xi)$, of distributions on $\Xi$ such that

$$
T_{b}=\left(d_{b} / d_{a}\right)^{s} T_{a} .
$$

We define a function $\hat{T}$ on $X$ for each $T$ in $\mathcal{B}_{s}(\Sigma)$ by $\hat{T}(a)=\left\langle T_{a}, 1\right\rangle$.

We prove that the map $T \mapsto \hat{T}$ is an isomorphism from $\mathcal{B}_{s}(\Xi)$ onto the eigenspace associated to the eigenvalue $\lambda$ corresponding to $s$ if the character $s$ is not singular. For $f$ an eigenfunction with eigenvalue $\lambda$, there is a nonsingular character $s$ for which it is the corresponding eigenvalue, and the distribution $T_{a}$ is given by an explicit formula

$$
\left\langle T_{a}, \varphi\right\rangle=\int_{\Xi}\left(r_{n-1}^{a} q_{a, n} f_{a, n+1}-r_{n}^{a} f_{a, n}\right) \varphi d_{a}
$$

if $\varphi$ factors through $\Xi \rightarrow S(a, n)$, where $r_{n}^{a}$ is an explicit sequence, depending on the character $s$ (see Theorems 3.2 and 3.9).

An Australina Research Council grant partially supports the visit of Gerardin in April 1997 during which this paper was written.

\section{Semi-homogeneous tree.}

1.1. . A tree is a connected graph without circuits. The number of edges to which a vertex $x$ of a tree belongs is called the valency of $x$. Given two integers $q_{-}, q_{+}$such that $1 \leq q_{-}<q_{+}$, we say $X$ is a semi-homogeneous tree of type $\left(q_{-}, q_{+}\right)$if every vertex has valency $q_{-}+1$ or $q_{+}+1$ and two adjacent vertices have different valencies. For $x$ in $X$ we write $q(x)=q_{-}$ (resp. $q_{+}$) if the valency of $x$ is $q_{-}+1$ (resp. $q_{+}+1$ ). This gives the degree function $q: X \rightarrow\left\{q_{-}, q_{+}\right\}$of $X$.

Let $F$ be a nonarchimedean local field, $q$ be the order of its residue field, $E / F$ be an unramified quadratic extension. Then the Bruhat-Tits building of the special unitary group over $F$ with respect to the hermitian form $\bar{x}_{0} y_{0}+\bar{x}_{1} y_{2}+\bar{x}_{2} y_{1}$ is a semi-homogeneous tree of type $\left(q, q^{3}\right)$. (Here: $x \mapsto \bar{x}$ denotes the non trivial automorphism of $E / F$.)

Given any two vertices $x, y$ in $X$ there exists a unique sequence $x_{0}, \ldots, x_{n}$ of vertices in $X$ such that $x=x_{0}, x_{i}$ is adjacent to $x_{i+1}$ for $0 \leq i \leq n-1$, $y=x_{n}$. The sequence $x_{0}, \ldots, x_{n}$ is also denoted by $[x, y]$. The integer $n$ is called the distance, $d(x, y)$, between $x$ and $y$. Given $a$ in $X$, the set $S(a, n)$ of vertices $x \in X$ at distance $n$ from $a$ is called the sphere at $a$ of radius $n$. If we write $q_{n}(a)$ for $q\left(a_{n}\right)$ with $a_{n} \in S(a, n)$, then the cardinality of $S(a, n)$ is, for $n \geq 1$ :

$$
|S(a, n)|=(1+q(a)) q_{1}(a) \cdots q_{n-1}(a) .
$$

We shall write $(a)_{n}$ for $|S(a, n+1)| /\left(1+q_{n}(a)\right), n \geq 0$. We define $(b: a)$ to be 1 for $a=b$ and to be $|S(a, n)|\left(1+q(b)^{-1}\right)^{-1}$ for $d(a, b)=n>1$. 
1.2. . The set consisting of connected components of complements of nonempty finite subsets of $X$ forms a projective system; its inverse limit is called the boundary of $X$ and it is a profinite compact space. We denote the boundary by $\Xi$. For each $a \in X$ we can identify $\Xi$ with the inverse limit $\varliminf_{n \geq 0} S(a, n)$ with respect to the projections $S(a, n+1) \rightarrow S(a, n)$.

For two distinct vertices $a, c$ in $X$ we write $X(c, \check{a})$ for the half-tree of $X$ with vertex at $c$ and not containing $a$ i.e., $X(c, \check{a})=\{x \in X \mid c \in(a, x]\}$. The boundary of $X(c, \check{a})$, denoted by $\Xi(c, \check{a})$, is the set of $\xi$ in $\Xi$ such that $c$ is in $(a, \xi)$. The sets $\Xi(c, \check{a})$ form a base of open sets for the compact space $\Xi$.

For $a \in X$, let $d_{a}$ be the probability measure on $\Xi$ such that

$$
\int_{\Xi(c, \breve{a})} d_{a} \xi=|S(a, n)|^{-1}
$$

for $c \in S(a, n)$, with $n \geq 1$. The space $\mathcal{D}(\Xi)$ of test functions on the boundary $\Xi$ is the space of locally constant functions on the compact space $\Xi$. For a vertex $a$ in $X$, the characteristic functions of $\Xi(c, \check{a})$ generate linearly $\mathcal{D}(\Xi)$. Let also $\mathcal{D}_{a, n}(\Xi)$ be the subspace of $\mathcal{D}(\Xi)$ consisting of those functions which factor through $S(a, n), n \geq 0$. These spaces $\mathcal{D}_{a, n}(\Xi)$, for $a$ fixed, are increasing in $n$ and $\mathcal{D}(\Xi)$ is the union of $\mathcal{D}_{a, n}(\Xi)$. The $a$-level of $\varphi \in \mathcal{D}(\Xi)$ is defined to be the largest integer $n$ such that $\varphi \in \mathcal{D}_{a, n}(\Xi)$. We denote by $\mathcal{D}^{\prime}(\Xi)$ the space of distributions on $\Xi$ and this is the inverse limit of finite dimensional spaces over $\mathbb{C}$. For example, for each vertex $a \in X$, it is the inverse limit of the dual spaces $\mathcal{D}_{a, n}^{\prime}(\Xi)$ of the above spaces $\mathcal{D}_{a, n}(\Xi)$.

Let $X_{+}$(resp. $X_{-}$) be the set of vertices in $X$ with degree $q_{+}$(resp. $q_{-}$). A complex function $f$ on $X$ invariant under the automorphisms of $X$ has constant value on $X_{+}$and on $X_{-}$; we write simply $f\left(X_{+}\right), f\left(X_{-}\right)$for these values. The algebra $\mathbb{C}[q]$ of functions on $X$ invariant under any automorphism of $X$ is isomorphic to the algebra $\mathbb{C} \times \mathbb{C}$ by $f \mapsto\left(f\left(X_{+}\right), f\left(X_{-}\right)\right)$, and has a corresponding involution $f \mapsto \bar{f}$ given by $\bar{f}\left(X_{+}\right)=f\left(X_{-}\right), \bar{f}\left(X_{-}\right)=f\left(X_{+}\right)$; in particular this defines $\bar{q}$. The algebra $\mathbb{C}[q]$ is defined by $\left(q-q_{+}\right)\left(q-q_{-}\right)=$ 0 .

Let $\mathcal{E}(X)$ be the algebra of all complex functions on $X$ and this is $\mathcal{E}\left(X_{+}\right) \times$ $\mathcal{E}\left(X_{-}\right)$, where $\mathcal{E}\left(X_{+}\right)$(resp. $\mathcal{E}\left(X_{-}\right)$) is defined similarly.

Given a vertex $a$ and an integer $n \geq 0$, we define an algebra homomorphism from $\mathcal{E}(X)$ to $\mathcal{D}_{a, n}(\Xi)$ as follows. For $f$ in $\mathcal{E}(X)$, let $f_{a . n}$ be the pullback to the boundary $\Xi$ of the restriction of $f$ to $S(a, n)$, i.e., for $x \in S(a, n)$ and $\xi \in \Xi(x, \check{a}), f_{a, n}(\xi)=f(x)$.

1.3. . For each $n \geq 0$, let $\Delta_{n}$ be the average operator on $\mathcal{E}(X)$ over spheres of radius $n$ : for $f \in \mathcal{E}(X),\left(\Delta_{n} f\right)(a)=\int_{\Xi} f_{a, n}(\xi) d_{a} \xi=\frac{1}{|S(a, n)|} \sum_{t \in S(a, n)} f(t)$. Write $\Delta$ for $\Delta_{1}$. 


\section{Lemma 1.4.}

(1) For $c \in \mathbb{C}[q]$, we have $\Delta c=\bar{c} \Delta$.

(2) $(\bar{q}+1) \Delta \Delta_{n}=\bar{q} \Delta_{n+1}+\Delta_{n-1}, n \geq 1$.

(3) $\left(q_{n}+1\right) \Delta_{n} \Delta=q_{n} \Delta_{n+1}+\Delta_{n-1}, n \geq 1$.

Proof. We prove (2). For a function $f$ on $X$ we have

$$
\left(\Delta \Delta_{n} f\right)(a)=\frac{1}{|S(a, 1)|} \sum_{t \in S(a, 1)}\left(\frac{1}{|S(t, n)|} \sum_{z \in S(t, n)} f(z)\right) .
$$

Note that $S(t, n)=\{u \in S(a, n+1) \mid t \in[a, u]\} \cup\{v \in S(a, n-1) \mid x \in[t, v]\}$, and each $u \in S(a, n+1)$ corresponds to a unique $t \in S(a, 1)$ such that $t \in[a, u]$, while each $v \in S(a, n-1)$ corresponds to $q(a)$ elements $t$ in $S(a, 1)$ such that $a \in[t, v]$. Thus

$$
\begin{aligned}
\left(\Delta \Delta_{n} f\right)(a)= & \frac{1}{q(a)+1} \frac{1}{|S(t, n)|} \sum_{S(a, n+1)} f(u) \\
& +\frac{q(a)}{q(a)+1} \frac{1}{|S(t, n)|} \sum_{S(a, n-1)} f(v) .
\end{aligned}
$$

Hence,

$$
\begin{aligned}
\Delta_{n} & =\frac{1}{q(a)+1} \frac{|S(a, n+1)|}{|S(t, n)|} \Delta_{n+1}+\frac{q(a)}{q(a)+1} \frac{|S(a, n-1)|}{|S(t, n)|} \Delta_{n-1} \\
& =\frac{q(t)}{q(t)+1} \Delta_{n+1}+\frac{1}{q(t)+1} \Delta_{n-1} .
\end{aligned}
$$

The proof is completed.

\section{Characters of semi-homogeneous trees.}

2.1. . Given vertices $a, b$ in $X$ the Radon-Nikodym cocycle is the function on $\Xi$ such that

$$
d_{b} \xi / d_{a} \xi=(b: a)_{\xi}, \quad \xi \in \Xi .
$$

We write $[a, \xi)$ for the half-line starting at $a$ in the direction of $\xi$. If $b \in[a, \xi)$ then

$$
(b: a)_{\xi}=\frac{1+q(a)}{1+q(b)} q_{1}(a) \cdots q_{d(a, b)}(a)=(b: a) ;
$$

if $a \in[b, \xi)$ then

$$
(b: a)_{\xi}=\frac{1+q(a)}{1+q(b)}\left(q_{1}(b) \cdots q_{d(a, b)}(b)\right)^{-1}=(a: b)^{-1}
$$

and in general,

$$
(b: a)_{\xi}=(b: c)_{\xi} /(a: c)_{\xi}=(c: a) /(c: b)
$$


where $c$ is any vertex common to $[a, \xi)$ and $[b, \xi)$.

The invariant function $\frac{1+q}{1+\bar{q}^{-1}}$ takes two distinct values, and its norm is $q \bar{q}=q_{+} q_{-}$, denoted by $Q$. The elements of the set $(X: X)_{\Xi}$ of values $(b: a)_{\xi}$, where $a, b$ are vertices of $X$ and $\xi$ is a boundary point, are either integral powers of $Q$, or the products of some integral power of $Q$ with one of the two values of $\frac{1+q}{1+\bar{q}^{-1}}$.

By a character of the semi-homogeneous tree $X$ with degree map $q$, we mean a map $s$ from this set $(X: X)_{\Xi}$ to the group $\mathbb{C}^{\times}$, written $(b: a)_{\xi} \mapsto(b$ : $a)_{\xi}^{s}$, such that the following identity holds: $(b: a)_{\xi}^{s}=(b: c)_{\xi}^{s}(c: a)_{\xi}^{s}$. The character $s$ is completely determined by the values of the invariant function $\left(\frac{1+q}{1+\bar{q}^{-1}}\right)^{s}$, and this gives an isomorphism from the group $\Sigma(X)$ of characters of $X$ with the multiplicative group $\mathbb{C}[q]^{\times}$of nonvanishing invariant functions, that is, with the product of two copies of $\mathbb{C}^{\times}$. A character $s$ of $X$ is called singular if $Q^{s}=1$. This means that its corresponding invariant function has norm 1 , and this defines an isomorphism from the group $\Sigma(X)^{1}$ of singular characters of $X$ onto the group $\mathbb{C}[q]^{1}$ of norm 1 invariant functions.

Given a character $s$ of $X$, we introduce the corresponding eigenvalue function $\lambda_{n}(a)$ by:

$$
\lambda_{n}(a)=\oint_{t \in S(a, n)}(t: a)_{\xi}^{s}
$$

where $\oint_{S(a, n)}$ is $\frac{1}{|S(a, n)|} \sum_{t \in S(a, n)}$. We write $\lambda$ for $\lambda_{1}$. That $\lambda_{n}(a)$ is independent of the choice of $\xi$ in $\Xi$ follows from

(1) for $a \in X$, the stabilizer $\operatorname{Aut}(X)_{a}$ of $a$ in the automorphism group $\operatorname{Aut}(X)$ of $X$ acts transitively on $\Xi$,

(2) $S(a, n)$ is an orbit of $\operatorname{Aut}(X)_{a}$ and

(3) $(g b: g a)_{g \xi}=(b: a)_{\xi}$ for any $g$ in $\operatorname{Aut}(X)$.

\section{Lemma 2.2.}

(1) $\lambda_{1}=\left(\frac{1+q}{1+\bar{q}^{-1}}\right)^{s} \frac{q Q^{-s}+1}{q+1}$.

(2) $\lambda_{n+1}(a)=\left(a_{n-1}: a_{n}\right)_{\xi}^{s} \lambda_{n}(a)+\frac{\left(a_{n+1}: a_{n}\right)_{\xi}^{s}-\left(a_{n-1}: a_{n}\right)_{\xi}^{s}}{1+q\left(a_{n}\right)}\left(a_{n}: a\right)_{\xi}^{s}$

(here $a_{n}$ is the vertex on $[a, \xi)$ at distance $n$ from $a$ ).

Proof. By definition

$$
\lambda_{1}(a)=\frac{1}{|S(a, 1)|} \sum_{t \in S(a, 1)}(t: a)_{\xi}^{s} .
$$

The sum over $S(a, 1)$ consists of the vertex $a_{1}$ on $[a, \xi)$ and $q(a)$ other vertices $a_{-1}$ in $S(a, 1)$.

$$
\lambda_{1}(a)=\frac{1}{1+q(a)}\left(\left(a_{1}: a\right)_{\xi}^{s}+q(a)\left(a_{-1}: x\right)_{\xi}^{s}\right) .
$$


The formula follows from $\left(a_{1}: a\right)_{\xi}=\bar{q}(a)\left(1+q(a)(1+\bar{q}(a))^{-1}\right.$ and $\left(a_{-1}\right.$ : $a)_{\xi}=(1+q(a)) q(a)(1+\bar{q}(a))^{-1}$. This proves (1). The proof of (2) is similiar.

2.3. . Given a character $s \in \Sigma(X)$ with associated invariant function $\lambda$, the space of eigenfunctions of the average operator $\Delta$ is denoted by $\mathcal{E}_{s}(X)$

$$
\mathcal{E}_{s}(X)=\{f \in \mathcal{E}(X) \mid \Delta f=\lambda f\} .
$$

The elements of $\mathcal{E}_{s}(X)$ for which the eigenvalue $\lambda$ is 1 are called harmonic functions. If $f \in \mathcal{E}_{s}(X)$ then $\Delta_{n} f=\lambda_{n} f$. The function $x \mapsto(x: a)_{\xi}^{s}$ is in $\mathcal{E}_{s}(X)$ for fixed vertex $a$ in $X$ and $\xi$ in the boundary $\Xi$.

2.4. . For a given character $s \in \Sigma(X)$, let $\lambda$ be the corresponding eigenvalue. The map $s \mapsto \lambda$ from $\Sigma(X)$ to $\mathbb{C}[q]$ has the following properties:

a) its image consists of all the nonzero elements of $\mathbb{C}[q]$;

b) the map $s \mapsto \lambda$ is one-to-one on each of the three following subsets of $\Sigma(X): Q^{s}=-q_{+}, Q^{s}=-q_{-}, Q^{2 s}=Q$

c) otherwise this map is two-to-one, the two distinct characters $s$ and $s^{*}$ giving the same eigenvalue if and only if

$$
Q^{s} Q^{s^{*}}=Q \text { and }\left(\frac{1+q_{+}}{1+q_{-}^{-1}}\right)^{s-s^{*}}=\frac{1+q_{+} Q^{-s}}{1+q_{+} Q^{-s^{*}}}
$$

(in fact, for each nonzero $\lambda$, its norm $\lambda \bar{\lambda}$ is $\frac{q_{+}+q_{-}+Q^{s}+Q^{1-s}}{\left(q_{+}+1\right)\left(q_{-}+1\right)}$, which is a degree two equation in $Q^{s}$ ):

d) the eigenvalue $\lambda$ has norm 1 if and only if $s$ or $1-s$ is singular, and the map $s \mapsto \lambda$ is an isomorphism from the group $\Sigma(X)^{1}$ of singular characters onto the group $\mathbb{C}[q]^{1}$ of norm 1 invariant functions;

e) finally, given $\lambda \neq 0$, there is always a nonsingular character $s$ with eigenvalue $\lambda$.

2.5. . For a character $s$ of $X$, let $\psi_{s}$ be the function on $X \times X$ given by

$$
\psi_{s}(a, b)=\int_{\Xi}(b: a)_{\xi}^{s} d_{a} \xi
$$

From $d_{b} \xi=(b: a)_{\xi} d_{a} \xi$, it follows that the function associated to the character $1-s$ is obtained from the one associated to $s$ by permuting the variables. For each $c$ in $[a, b]$, the integrand is constant on $\Xi(c, \check{a}) \cap \Xi(c, \breve{b})$ (with the convention $\Xi(x, \check{x})=\Xi$ ). These subsets form a partition of $\Xi$, and $\psi_{s}(a, b)$ appears as a finite sum, showing it as a regular function on $\Sigma(X)$. The number $\psi_{s}(a, b)$ depends only on the orbit of $(a, b)$ under the automorphism group of $X$ since $(g b: g a)_{g \xi}=(b: a)_{\xi}$. These functions $\psi_{s}(a, b)$ are the zonal invariant kernels on the space $X$. 
Proposition 2.6. Let $s$ be a character of $X$ and $\lambda_{n}$ be the corresponding invariant eigenvalue of index $n$. Given two vertices $a, b$ of $X$, then

(1) $\psi_{s}(a, b)=\lambda_{n}(a)$ if $d(a, b)=n$,

(2) each function $x \mapsto \psi_{s}(a, x)$ lies in the eigenspace $\mathcal{E}_{s}(X)$,

(3) if $\alpha, \beta \in \Xi$ satisfy $a \in[b, \beta), b \in[b, \alpha)$, then the series $\sum(y: a)_{\alpha}^{s}$ over those $y \in X$ such that $(y: b)_{\beta}=1$ converges for $\left|Q^{s}\right|<Q^{1 / 2}$, and its sum is $\left(1+q(b)^{-1}\right) c(b, s)(b: a)^{s}$,

(4) $\psi_{s}(a, b)=c(b, s)(b: a)^{s}+c(a, 1-s)(a: b)^{1-s}$ where $c(x, s)$ is the following rational function on $\Sigma(X) / \Sigma(X)^{1}$ :

$$
c(x, s)=\frac{1-Q^{-s}}{1-Q^{1-2 s}} \frac{1+q(x)^{-1} Q^{1-s}}{1+q(x)^{-1}} .
$$

The proof consists of a direct calculation which we shall omit.

\section{Boundary values of eigenfunctions.}

3.1. . Each eigenfunction $f$ on the tree $X$ will have a boundary value which is a section of a suitable bundle of distributions over $\Xi$ with base $X$. For each vertex $a$ of $X$, the corresponding distribution on $\Xi$ will appear as a limit, in the topology of $\mathcal{D}^{\prime}(\Xi)$, of a linear combination of the distributions $f_{a, n} d_{a}$ and $f_{a, n+1} d_{a}$. More precisely, the following theorem gives explicitly the projection of this boundary distribution on the space $\mathcal{D}^{\prime}(\Xi)$.

Given a nonsingular character $s$ of $X$, we define the following sequence, depending on $s$ :

$$
r_{n}^{a}=|S(a, n+1)| /\left((a)_{n+2}^{s}-(a)_{n}^{s}\right), n \geq 0 .
$$

Theorem 3.2. Let $X$ be a semi-homogeneous tree with degree function $q$, $s$ be a nonsingular character of $X$ and $f \in \mathcal{E}_{s}(X)$.

(1) For each $a$ in $X$ and $\varphi$ in $\mathcal{D}(\Xi)$, the number

$$
\int_{\Xi}\left(r_{n-1}^{a} q_{a, n} f_{a, n+1}-r_{n}^{a} f_{a, n}\right) \varphi d_{a}
$$

is independent of $n \geq$ a-level of $\varphi$, and defines a distribution $\hat{f}_{a}$ on $\Xi$ for which $f(a)=\left\langle\hat{f}_{a}, 1\right\rangle$;

(2) for $\xi_{0}$ in $\Xi$ and $f$ the function $x \mapsto(x: a)_{\xi_{0}}^{s}$, this distribution is the Dirac measure $\delta_{\xi_{0}}$ at $\xi_{0}$;

(3) for $a$ and $b$ in $X$, the distributions $\hat{f}_{a}$ and $\hat{f}_{b}$ are related by the formula $\hat{f}_{b}(\xi)=(b: a)_{\xi}^{s} \hat{f}_{a}(\xi)$.

Remark. The formula in (1) reduces to the earlier formula for homogeneous trees as stated in $[\mathbf{G}] \S 2.4$ when we put $q_{-}=q_{+}$. See Cohen-Colonna $[\mathbf{C C}]$ Proposition 1 for the case of a homogeneous tree. 
Proof.

I. We apply the formula $\Delta f=\lambda f$ at each point $a_{n}$ of the sphere $S(a, n), n \geq$ 1:

$$
\left(1+q\left(a_{n}\right)\right) \lambda\left(a_{n}\right) f\left(a_{n}\right)=\sum_{\substack{t \in S\left(a_{n}, 1\right) \\ t \neq a_{n}-1}} f(t)+f\left(a_{n-1}\right),
$$

where $a_{n-1}$ is the neighbour of $a_{n}$ which belongs to $S(a, n-1)$. Now let $\varphi$ be a test function on $\Xi$ with $a$-level at most $n$; we write also $\varphi$ for the functions on $S(a, m), m \geq n$, coming from it. We have, using the constant function $q_{a, n}$ on $S(a, n)$ :

$$
\int_{\Xi} f_{a, n+1} \varphi d_{a}=\oint_{S(a, n+1)} f_{a, n+1} \varphi=\frac{1}{q_{a, n}} \oint_{S(a, n)} \sum f\left(a_{n+1}\right) \varphi
$$

where the inner sum is on all $a_{n+1}$ in $S(a, n+1)$ coming from a common element in $S(a, n)$. The above formula can be rewritten as

$q_{a, n} \int_{\Xi} f_{a, n+1} \varphi=\frac{1}{|S(a, n)|} \sum_{a_{n} \in S(a, n)}\left(\left(1+q\left(a_{n}\right)\right) \lambda\left(a_{n}\right) f\left(a_{n}\right)-f\left(a_{n-1}\right)\right) \varphi\left(a_{n}\right)$.

If, moreover, the $a$-level of $\varphi$ is at most $n-1$, then the right hand side is simply

$$
\int_{\Xi}\left(\left(1+q_{a, n}\right) \lambda_{a, n} f_{a, n}-f_{a, n-1}\right) \varphi d_{a} .
$$

Next from $\oint_{S\left(a_{n, 1}\right)}(x: a)_{\xi}^{s}=\lambda\left(a_{n}\right)(a)_{n}^{s}=\lambda_{a, n}(a)_{n}^{s}$, we obtain

$$
\left(1+q_{a, n}\right) \lambda_{a, n}(a)_{n}^{s}=q_{a, n}(a)_{n-1}^{s}+(a)_{n+1}^{s}
$$

so that the function

$$
q_{a . n} f_{a, n+1}-\left(1+q_{a, n}\right) \lambda_{a, n} f_{a, n}+f_{a, n-1}
$$

is also

$$
q_{a, n}\left(f_{a, n+1}-\frac{(a)_{n-1}^{s}}{(a)_{n}^{s}} f_{a, n}\right)-\left(\frac{(a)_{n+1}^{s}}{(a)_{n}^{s}} f_{a, n}-f_{a, n-1}\right)
$$

which is

$$
\begin{aligned}
\frac{(a)_{n-1}^{s}}{|S(a . n)|}\left(|S(a, n+1)|\left(Q^{s} \frac{f_{a, n+1}}{(a)_{n+1}^{s}}-\frac{f_{a, n}}{(a)_{n}^{s}}\right)\right. & \\
& \left.-|S(a, n)|\left(Q^{s} \frac{f_{a, n}}{(a)_{n}^{s}}-\frac{f_{a, n-1}}{(a)_{n-1}^{s}}\right)\right)
\end{aligned}
$$

since $\left(a_{n+1}: a_{n-1}\right)=Q$ and $|S(a, n+1)|=q_{a, n}|S(a, n)|$. So, we have shown that given $\varphi \in \mathcal{D}(\Xi)$, then

$$
\left\langle|S(a, n+1)|\left(Q^{s} \frac{f_{a, n+1}}{(a)_{n+1}^{s}}-\frac{f_{a, n}}{(a)_{n}^{s}}\right) d_{a}, \varphi\right\rangle
$$


for all $n \geq$ the level of $\varphi$ from $a$ has the same value. We have found a candidate for the weighted average that satisfies our stability requirement.

We apply this result to the test function $\varphi=1$, which has level 0 with respect to any vertex of $X$; so, for $n=0$, we get

$$
\begin{aligned}
& \left\langle|S(a, 1)|\left(Q^{s} \frac{f_{a, 1}}{(a)_{1}^{s}}-f(a)\right) d_{a}, 1\right\rangle \\
& =|S(a, 1)|\left(Q^{s} \frac{\lambda(a)}{(a)_{1}^{s}} f(a)-f(a)\right) \\
& =\left(Q^{s}-1\right) f(a) .
\end{aligned}
$$

We have used

$$
\lambda(a)=\oint_{t \in S(a, 1)}(t: a)_{\xi}^{s}=\frac{q(a)\left(a_{-1}: a\right)_{\xi}^{s}+\left(a_{1}: a\right)_{\xi}^{s}}{q(a)+1}=\frac{q(a) Q^{-s}+1}{q(a)+1}(a)_{1}^{s}
$$

(see Lemma 2.2).

This calculation shows that our choice of $r_{n}^{a}$ has the correct normalization required by the condition $\left\langle\hat{f}_{a}, 1\right\rangle=f(a)$.

We have proved part (1) of the theorem.

II. In this part we put $f(x)=(x: a)_{\xi_{0}}^{s}$ with $a$ in the tree $X$ and $\xi_{0}$ in the boundary $\Xi$. For $c \neq a$, let

$$
V(c)=\int_{\Xi(c, \breve{a})}\left(r_{n-1}^{a} q_{a, n} f_{a, n+1}-r_{n}^{a} f_{a, n}\right) d_{a} .
$$

It follows from part I of the proof that this is independent of $n \geq d(a, c)$.

Lemma 3.3. If $c \notin\left[a, \xi_{0}\right)$ and $a \in\left[c, \xi_{0}\right)$ then $V(c)=0$.

Proof. First assume $d(c, a)=1$. Then we take $n=1$ and we get

$$
\begin{aligned}
V(c) & =r_{0}^{a} \bar{q}(a) \int_{\Xi(c, \breve{a})} f_{a, 2} d_{a}-r_{1}^{a} \int_{\Xi(c, \breve{a})} f_{a, 1} d_{a} \\
& =r_{0}^{a} \bar{q}(a) \oint_{S(a, 2) \cap X(c, \breve{a})} f(y)-r_{1}^{a} \oint_{S(a, 1) \cap X(c, \breve{a})} f(x) \\
& =r_{0}^{a} \bar{q}(a) \frac{\bar{q}(a)}{(1+q(a)) \bar{q}(a)}\left(c_{1}: a\right)_{\xi_{0}}^{s}-r_{1}^{a} \frac{(c: a)_{\xi_{0}}^{s}}{1+q(a)} \\
& =0 .
\end{aligned}
$$

Next suppose that $d(c, a)>1$. On $S(a, n) \cap X(c, \check{a})$ our function $f$ is constant. Hence

$$
V(c)=\sum_{c^{\prime} \in S(a, n) \cap X(c, \check{a})} V\left(c^{\prime}\right)=|S(a, n) \cap X(c, \check{a})| V\left(c^{\prime}\right)
$$

and $V\left(c^{\prime}\right)=0$ follow from $V(c)=0$ by induction on $d(c, a)$.

Lemma 3.4. If $c \notin\left[a, \xi_{0}\right)$ and $a \notin\left[c, \xi_{0}\right)$ then $V(c)=0$. 
Proof. Take a vertex $b$ in $\left[a, \xi_{0}\right) \cap\left[c, \xi_{0}\right)$. Choose positive integers $m, n$ such that $m+d(a, b)=n$. Then for our function $f$, we have

$$
f_{a, n}=(b: a)_{\xi_{0}}^{s} f_{b, m} .
$$

It follows from the definition of $r$ that

$$
r_{m}^{b}=(b: a)_{\xi_{0}}^{s-1} r_{n}^{a} .
$$

If we write $V(c, a, n)$ for $V(c)$ to indicate the role of $a$ and $n$, then the above remarks imply that

$$
V(c, a, n)=V(c, b, m), m+d(a, b)=n .
$$

By the previous Lemma 3.3 we have $V(c, b, m)=0$, so $V(c, a, n)=0$.

Lemma 3.5. If $c \in\left(a, \xi_{0}\right)$ then $V(c)=1$.

Proof. Since $\Xi$ is the disjoint union of $\Xi\left(c^{\prime}, \check{a}\right)$ with $c^{\prime} \in S(a, n), n=d(a, c)$, by the previous two lemmas we have

$$
V(c)=\int_{\Xi} \hat{f}_{a}=(a: a)_{\xi_{0}}^{s}=1 .
$$

These lemmas yield the proof of condition (2) of the theorem.

III. We only have to verify condition (3) on a test function with support in a small neighbourhood of a point $\xi_{0}$ in the boundary $\Xi$. Take a vertex $c_{0}$ in $\left(a, \xi_{0}\right) \cap\left(b, \xi_{0}\right)$. Take a vertex $c$ in $\left(c_{0}, \xi_{0}\right)$ and a test function $\varphi$ supported in $\Xi(c, \check{a})=\Xi(c, \breve{b})$. Then $r_{m}^{b}=(b: a)_{\xi_{0}}^{s-1} r_{n}^{a}$ where $m=d(b, c), n=d(a, c)$. Condition (3) follows immediately.

Remark 3.6. Implicit in the proof of the theorem is the fact that $r_{n}^{a}$ is the solution of the following difference equation satisfied by the spherical functions:

$$
\lambda_{n}(a) X_{n}-q_{n}(a) \lambda_{n+1}(a) X_{n-1}+1=0, n \geq 1
$$

under the initial condition

$$
X_{0}=\frac{1+q(a)}{Q^{s}-1} .
$$

This is imposed by the normalization and stability conditions.

3.7. . Let $\mathcal{B}_{s}(\Xi)$ be the space of maps $T: X \rightarrow \mathcal{D}^{\prime}(\Xi): x \mapsto T_{x}$ with $T_{x}$ satisfying the condition:

$$
T_{y}(\xi)=(y: x)_{\xi}^{s} T_{x}(\xi) .
$$

For $T \in \mathcal{B}_{s}(\Xi)$, define $\hat{T}(x)=\left\langle T_{x}, 1\right\rangle$. For each fixed $a$ in $X$, the map $\mathcal{B}_{s}(\Xi) \rightarrow \mathcal{D}^{\prime}(\Xi)$ given by $T \mapsto T_{a}$ is an isomorphism.

Lemma 3.8. If $T \in \mathcal{B}_{s}(\Xi)$ then $\hat{T} \in \mathcal{E}_{s}(X)$. 
Proof. We have

$$
\begin{aligned}
\Delta \hat{T}(x) & =\oint_{y \in S(x, 1)} \hat{T}(y)=\oint_{y \in S(x, 1)} \int_{\Xi} T_{y} \\
& =\int_{\Xi} \oint_{y \in S(x, 1)}(y: x)_{\xi}^{s} T_{x}=\lambda(x) \hat{T}(x) .
\end{aligned}
$$

On the other hand, we have seen that from $f \in \mathcal{E}_{s}(X)$ we get $\hat{f} \in \mathcal{B}_{s}(\Xi)$, namely, $\hat{f}: x \mapsto \hat{f}_{x}$.

Theorem 3.9. Let $X$ be a semi-homogenenous tree with degree function $q$. Let $s$ be a nonsingular character of $X$. Then the map

$$
\mathcal{B}_{s}(\Xi) \longrightarrow \mathcal{E}_{s}(X)
$$

which sends $T$ to the function $\hat{T}(x)=\left\langle T_{x}, 1\right\rangle$ is an isomorphism.

Proof. Start with $T \in \mathcal{B}_{s}(\Xi)$ we obtain $\hat{T} \in \mathcal{E}_{s}(X)$. We claim that $\hat{T}(a)=T_{a}$ for all vertices $a$. We only have to evaluate these distributions on test functions of sufficiently small support. We observe that $\hat{T}(x)=\langle(x$ : $\left.a)_{\xi^{\prime}}^{s} T_{a}\left(\xi^{\prime}\right), 1\right\rangle$. Take $\varphi \in \mathcal{D}_{a, n}(\Xi)$. Then

$$
\left\langle(\hat{T})_{a}, \varphi\right\rangle=\int_{\Xi \times \Xi}\left(r_{n-1}^{a} q_{a, n}\left(x_{n+1}: a\right)_{\xi^{\prime}}^{s}-r_{n}^{a}\left(x_{n}: a\right)_{\xi^{\prime}}^{s}\right) T_{a}\left(\xi^{\prime}\right) \varphi(\xi) d_{a} \xi .
$$

For the function $f(x)=(x: a)_{\xi^{\prime}}^{s}$, we have $\hat{f}_{a}=\delta_{\xi^{\prime}}$ and so it follows that $\left\langle(\hat{T})_{a}, \varphi\right\rangle=\left\langle T_{a}, \varphi\right\rangle$. Conversely, given a function $f$ in $\mathcal{E}_{s}(X)$ we have, from Theorem 3.2, $\hat{f} \in \mathcal{B}_{s}(\Xi)$ and we have already shown that $\left\langle\hat{f}_{a}, 1\right\rangle=f(a)$.

3.10. . Assume now that $s$ is a singular character of $X$. Then, for any $\xi \in \Xi$, we have

$$
(b: a)_{\xi}^{s}=1 \text { if } q(a)=q(b),(b: a)_{\xi}^{s}=\left(\frac{1+q(a)}{1+\bar{q}(a)^{-1}}\right)^{s} \text { if } q(a) \neq q(b) .
$$

This implies that the image $f$ in $\mathcal{E}_{s}(X)$ of a given $T$ in $\mathcal{B}_{s}(\Xi)$ is determined by its value in a point $a \in X$, and satisfies:

$$
\begin{aligned}
& f(x)=f(a) \text { if } q(x)=q(a), \\
& f(x)=\left(\frac{1+q(a)}{1+\bar{q}(a)^{-1}}\right)^{s} \text { if } q(x) \neq q(a) .
\end{aligned}
$$

Theorem 3.11. Let $X$ be a semi-homogeneous tree with degree function $q$. Let $s$ be a singular character of $X$. Then, the image of $\mathcal{B}_{s}(\Xi)$ in $\mathcal{E}_{s}(X)$ is $a$ one dimensional subspace, and the choice of any vertex $a \in X$ defines a basis of it as follows:

$$
\begin{aligned}
& x \mapsto 1 \text { for } q(x)=q(a), \\
& x \mapsto\left(\frac{1+q(a)}{1+\bar{q}(a)^{-1}}\right)^{s} \text { for } q(x) \neq q(a) .
\end{aligned}
$$


Remark. For a character $s$, the corresponding sequence $\lambda_{n}$ of eigenvalues satisfies: $\lambda_{n}=1$ for $n$ even, and $\lambda_{n}=\left(\frac{1+q}{1+\bar{q}^{-1}}\right)^{s}$ for $n$ odd. In particular, they all have norm equal to 1 . Conversely, if $\lambda_{1}$ has norm 1 , then Lemma 2.2 implies that $\lambda_{n}=1$ for $n$ even and $\lambda_{n}=\lambda_{1}$ for $n$ odd. The condition $\lambda_{1} \bar{\lambda}_{1}=1$ is equivalent to $Q^{s}=1$ or $Q^{s}=Q$, that is $s$ or $1-s$ is singular. If $s$ is singular then $1-s$ is not singular, and there is $s^{*}$ with same eigenvalue $\lambda$ as $s$, so that the spaces $\mathcal{E}_{s}(X)$ and $\mathcal{E}_{s *}(X)$ are the same, and $f \mapsto \hat{f}$ constructed above gives an isomorphism of $\mathcal{E}_{s}(X)=\mathcal{E}_{s *}(X)$ with $\mathcal{B}_{s *}(\Xi)$.

The result on harmonic functions on the unit disc (and its generalizations) can be found in $[\mathbf{H}] \S 4.1$ Theorem 4.3; Furstenberg [Fu], Kashiwara et. al. [Kas]. For asymptotic formula for harmonic functions on trees see Cohen-Colonna $[\mathbf{C C}]$. Harmonic analysis on semi-homogeneous trees has been studied by Bouaziz-Kellil $([\mathbf{B}])$, Choucroun $([\mathbf{C h}])$, Nevo $([\mathbf{N}])$ and Olshanskii $([\mathbf{O}])$. See also Kato ([Kat $])$. No asymptotic formula is given in these works. For further work on this problem for buildings of rank 2 see, for example, Cartwright and Mlotkowski [Car], Mantero and Zappa [MZ]; for buildings of type $A_{n}$ see Gérardin and Lai [GL].

\section{References}

[B] F. Bouaziz-Kallil, Représentations sphériques des groupes agissant transitivement sur un arbre semi-homogène, Bull. Soc. Math. France, 116 (1988), 255-278.

[C] P. Cartier, Fonctions harmoniques sur un arbre, Symps. Math., 9 (1972), 203-270.

[Ca] Harmonic analysis on trees, Proc. Symp. Pure Math., XXVI (1972), 419424.

[Car] D.I. Cartwright and W. Mlotkowski, Harmonic analysis for groups acting on triangle buildings, J. Austral. Math. Soc. (Series A), 56 (1994), 345-383.

[Ch] F. Choucroun, Analyse harmonique des groupes d'automorphismes d'arbres de Bruhat-Tits, Soc. Math. France, Mémoire 58, 1994.

[CC] Joel M. Cohen and F. Colonna, Spectral analysis on homogeneous trees, Adv. Applied Math., 20 (1998), 253-274.

[CP] Joel M. Cohen and L. De Michele, The radial Fourier Stieltjes algebra of free groups, Contemp. Math., 10 (1982), 33-40.

[F] A. Figà-Talamanca and C. Nebbia, Harmonic analysis and representation theory for groups acting on homogeneous trees, Cambridge University Press, 1991.

[FP] A. Figà-Talamanca and M.A. Picardello, Harmonic analysis on free groups, Dekker, New York, 1983.

[Fu] H. Furstenberg, A Poisson formula for semi-simple Lie groups, Ann. Math., 77 (1963), 335-386.

[G] P. Gérardin, On harmonic functions on symmetric spaces and buildings, Proceedings Canadian Math. Soc. Conference 1980, AMS, Providence, R.I.

[Ge] - Harmonic functions on buildings of reductive split groups, in 'Operator Algebra and Group Representations', Pitman, London, 1984. 
[GL] P. Gérardin and K. F. Lai, Opérateurs invariants sur les immeubles affines de type A, C.R. Acad. Sci. Paris, 329 (1999), 1-4.

[H] S. Helgason, Topics in Harmonic Analysis on Homogeneous Spaces, Birkhäuser, Boston, 1981.

[Kas] M. Kashiwara, A. Kowata, K. Minemura, K. Okamoto, T. Oshima and M. Tanaka, Eigenfunctions of invariant differential operators on a symmetric space, Ann. Math., 107 (1978), 1-39.

[Kat] S. Kato, On eigenspaces of the Hecke algebra with respect to a good maximal compact subgroup of a p-adic reductive group, Math. Ann., 257 (1981), 1-7.

[Ko] A. Koranyi (ed.), Harmonic functions on trees and buildings, Contemp. Math., 206, AMS, Providence R.I., 1997.

[M] A.M. Mantero and A. Zappa, The Poisson transform on free groups and uniformly bounded representations, J. Func. Ana., 51 (1983), 372-399.

[MZ] Spherical functions and spectrum of the Laplace operators on buildings of rank 2, Bollettino U. M. I., (7) 8-B (1994), 419-475.

$[\mathrm{N}]$ A. Nevo, Boundary theory and harmonic analysis on boundary transitive graphs, Am. J. Math., 116 (1994), 243-282.

[O] G. Olshanskii, Classification of irreducible representations of groups of automorphisms of Bruhat-Tits trees, Func. Anal. Appl., 11 (1977), 26-34.

[S] J.P. Serre, Arbres, Amalgames, SL $L_{2}$, Astérisque, 46 (1977).

[T] J. Tits, Sur le groupe des automorphismes d'un arbre, in 'Essay on Topology and related topics', Springer Verlag, (1970), 188-211.

Received February 10, 1999 and revised November 1, 1999.

C.N.R.S. Université PARIS VII

2, Place Jussieu

F-75251 PARIS CEDEX 5

FRANCE

School of Mathematics and Statistics

UNIVERSITY OF SYDNEY

N.S.W. 2006

Australia

E-mail address: kflai@maths.usyd.edu.au 